\title{
Simulador de Oscilações Mecânicas
}

\author{
Simulating Mechanical Oscillations
}

\author{
Adilson Costa da Silva*1, José Abdalla Helayël Neto² \\ ${ }^{1}$ Programa de Engenharia Nuclear, Universidade Federal do Rio de Janeiro, Rio de Janeiro, Brasil \\ ${ }^{2}$ Centro Brasileiro de Pesquisas Físicas, Urca, Rio de Janeiro, Brasil
}

Recebido em 17 de fevereiro de 2016. Aceito em 28 de março de 2016

\begin{abstract}
Recentemente desenvolvemos um software com o objetivo de proporcionar uma visão do fenômeno físico, sem a necessidade de conhecer a equação que descreve o movimento. Desta forma, este simulador visa ilustrar o comportamento das variáveis de interesse, tais como, posição, velocidade, aceleração e energia evoluindo no tempo. Neste artigo, apresentamos soluções analíticas para cada caso de interesse, bem como, soluções numéricas usando o método de diferenças finitas. Um oscilador harmônico representa um sistema em movimento a ser repetido ao longo do tempo, isto é, move-se de uma posição inicial até outra posição, em torno de uma posição de equilíbrio. Desta forma, apresentaremos uma visão geral do problema a ser resolvido e os casos mais simples serão obtidos por simplificações deste problema. Como um dos objetivos deste artigo é incentivar os leitores a utilizarem métodos numéricos de soluções aplicados à física, logo, abordamos de forma bastante simples a solução numérica do oscilador mecânico. Avaliamos também o comportamento da energia e verificamos sob quais condições ela é conservada. Para validar o método numérico implementado neste artigo, comparamos os resultados obtidos pela solução numérica com os resultados obtidos pela solução analítica usada neste artigo.

Palavras-chave: Oscilador harmônico mecânico, energia mecânica, forças externas, método de diferenças finitas e forças dissipativas.
\end{abstract}

We have recently developed a software to simulate a given physical phenomenon, without the need of knowing its corresponding equation of motion. This procedure is actually intended to illustrate the behavior of dynamical variables of interest, such as position, velocity, acceleration and energy. In our work, we present analytical solutions for each case under consideration; numerical solutions carried out by means of the finite difference method are presented as well. The harmonic oscillator is a moving periodical system, with a well-defined equilibrium position. We shall here present an overview of the general problem to be solved and a number of special cases shall be worked out in details. As one of the purposes of this article is to encourage readers to use numerical approaches to inspect physical problems, we shall discuss in a simple and direct way the numerical solutions of a mechanical oscillator. We also contemplate the behavior of the energy function and point out under which particular situations it is conserved. To validate the numerical method implemented in our paper, we compare the analytical solutions we derive with the results obtained by numerical computations.

Keywords: mechanical harmonic oscillator, mechanical energy, external forces, finite difference method and dissipative forces.

\section{Introdução}

Um dos principais objetivos da física é buscar modelos que visam descrever diferentes tipos de fenômenos físicos, bem como, experimentos em la-

\footnotetext{
*Endereço de correspondência: adilsoncostasilva@gmail.com
}

boratórios. Com isso, muitos pesquisadores buscam soluções analíticas para descrever esses fenômenos. No entanto, há problemas em aberto que não possuem soluções analíticas. Para contornar isso, muitos pesquisadores desenvolveram algoritmos que buscam 
resultados numéricos para modelar diferentes tipos de problemas, das mais diferentes áreas do conhecimento. Daí surgem diferentes tipos de métodos numéricos, da qual destacaremos o método de diferenças finitas [1]. Este método consiste em aproximar a derivada de uma função a partir de dois pontos consecutivos. Logo, quanto menor for o espaçamento entre esses pontos, mais preciso este método se torna, fazendo a solução numérica convergir para a solução analítica. No entanto, isso exige grande esforço computacional. Para ilustrar, considere um intervalo de tempo de $\Delta t=10^{-3} \mathrm{~s}$. Para determinar a posição de uma partícula no instante de tempo $t=1 s$, precisaríamos realizar mil cálculos usando este método. Porém, usando uma solução analítica realizaríamos apenas um único cálculo para determinar a posição dessa partícula neste mesmo instante de tempo.

O objetivo principal desse artigo é proporcionar aos leitores uma visão do problema físico além de permitir a implementação de métodos numéricos aplicados à física de forma bastante simples. Neste simulador buscamos ilustrar o comportamento das variáveis de interesse, tais como: posição, velocidade, aceleração, energia cinética, energia potencial e energia mecânica ao longo do tempo.

Apresentaremos uma visão geral do problema a ser resolvido e as abordagens mais simples serão obtidas por simplificações deste problema. Sendo assim, utilizaremos uma equação que descreva um oscilador harmônico amortecido sob a ação de uma força externa. Uma simplificação deste problema, consiste em assumir que não há forças externas atuando no sistema, logo, recaímos num sistema conhecido como oscilador harmônico amortecido, que pode ser classificado em: superamortecido, criticamente amortecido e subamortecido sob certas condições. Uma outra simplificação é assumirmos que não há forças dissipativas atuando no sistema, o que resulta em um oscilador harmônico simples, onde a energia do sistema é conservada.

Apesar do estudo de oscilações harmônicas serem para fins de resolução de sistemas de físicamatemática, suas aplicações se estender a várias áreas do conhecimento, tais como, movimentos que apresentam uma certa periodicidade ao longo do tempo, bem como, o estudo de fenômenos de ressonância em oscilações de pontes, materiais, circuitos elétricos entre outros.

Considere um bloco preso a uma mola que seja capaz de executar um movimento harmônico acoplado a uma haste em um meio viscoso, conforme ilustra a Fig. 1. Este sistema, por sua vez, está sob a ação de uma força externa que pode variar em função do tempo. Sendo assim, para determinar os parâmetros que descrevem este movimento, podemos fazer uso da $2^{a}$ lei de Newton, tal que,

$$
\mathbf{F}=m \mathbf{a},
$$

onde $\mathbf{F}$ representa todas as forças que atuam sobre o sistema e a é aceleração adquirida pelo bloco devido a ação dessas forças. Para simplificar, não usaremos a notação vetorial para força, aceleração, velocidade e posição, uma vez que, o movimento ocorrerá ao longo de uma única direção. Devido o sistema ser constituído por vários tipos de forças, iremos descrever brevemente cada uma delas. Logo, temos:

i) Força elástica $\left[F_{e l}(t)=-k x(t)\right]$ : É um tipo de força que surge quando uma mola desloca-se da posição de equilíbrio, onde $k$ é a constante elástica da mola.

ii) Força de resistência $\left[F_{r e}(t)=-b v(t)\right]$ : É um tipo de força que surge devido a resistência imposta pelo fluido ao movimento do bloco preso a haste, que por sua vez, amortece o sistema e converte a energia perdida pelo amortecimento em calor, onde b é a constante de amortecimento.

iii) Força externa $[F(t)]$ : É um tipo de força que atua externo ao sistema, com o objetivo de mudar as características do mesmo. Desta forma, esse tipo de força pode apresentar comportamentos distintos, ou seja, podem atuar de forma constante sobre o sistema ou simplesmente variar ao longo do tempo.

Com isso, usando a Eq. (1), podemos obter uma equação que descreve o comportamento desse bloco ao longo do tempo, tal que,

$$
F(t)-b v(t)-k x(t)=m a(t) .
$$

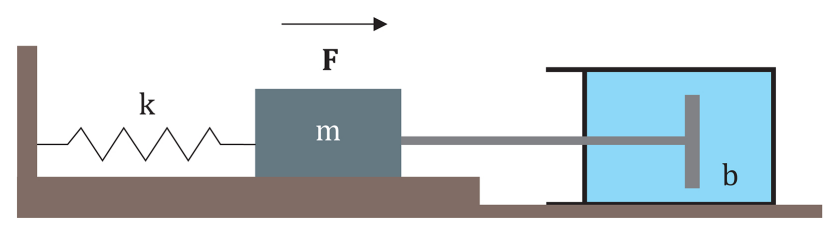

Figura 1: Oscilador harmônico amortecido forçado. 
Usando a notação de derivadas, onde a velocidade $v(t)=\frac{d x(t)}{d t}$ e a aceleração seja dada por, $a(t)=\frac{d^{2} x(t)}{d t^{2}}$, temos,

$$
m \frac{d^{2} x(t)}{d t^{2}}+b \frac{d x(t)}{d t}+k x(t)=F(t) .
$$

A Eq. (3) representa a formulação mais completa para descrever esse tipo de sistema. Para simplificar a abordagem que será feita, dividiremos este sistema em dois casos, segundo as técnicas utilizadas para resolução de equações diferenciais, qual seja

i) Oscilador harmônico amortecido: Neste caso, a força externa que atua sobre o sistema é nula. Com isso, temos uma equação homogênea dada por,

$$
m \frac{d^{2} x_{h}(t)}{d t^{2}}+b \frac{d x_{h}(t)}{d t}+k x_{h}(t)=0 .
$$

ii) Oscilador harmônico amortecido forçado: Neste caso, há uma força externa atuando no sistema cuja equação é dada por,

$$
m \frac{d^{2} x_{p}(t)}{d t^{2}}+b \frac{d x_{p}(t)}{d t}+k x_{p}(t)=F(t),
$$

A solução geral será dada pela combinação da solução homogênea $x_{h}(t)$ mais a particular $x_{p}(t)$, tal que

$$
x(t)=x_{h}(t)+x_{p}(t) .
$$

\section{Oscilador harmônico amortecido}

Apresentaremos a solução para o caso do oscilador harmônico amortecido. Neste caso, não há forças externas atuando sobre o sistema. Com isso, a equação é expressa da seguinte forma,

$$
m \frac{d^{2} x_{h}(t)}{d t^{2}}+b \frac{d x_{h}(t)}{d t}+k x_{h}(t)=0 .
$$

A técnica a ser utilizada para resolver essa equação diferencial, consiste em buscar uma função cuja as derivadas sejam expressas pela própria função, salvo de uma constante. Com isso, podemos descartar funções cujas derivadas alterem a forma ou o grau da função, tais como, funções polinomiais, logarítmica entre outras. No entanto, há funções que satisfazem esse tipo de condição, tais como, funções trigonométricas e exponenciais entre outras. Neste caso, usaremos funções exponenciais, dada por

\footnotetext{
${ }^{1}$ Onde $e^{i \theta}=\cos \theta+i \sin \theta$, onde $i^{2}=-1$.
}

$$
x_{h}(t)=A e^{i w t},
$$

onde $A$ é o coeficiente da solução que é obtido em função da condição inicial do sistema, $i$ a notação complexa associada a Equação de Euler 1], $w$ é o argumento da função exponencial e $t$ é o instante de tempo. Substituindo esta equação na Eq. (4), vem

$$
\left(m i^{2} w^{2}+b i w+k\right) x_{h}(t)=0,
$$

como $x_{h}(t)$ é a solução do sistema, logo, não pode ser nula, pois recairíamos na solução trivial. Desta forma, o termo em parênteses tem que ser nulo, ou seja,

$$
-m w^{2}+i b w+k=0
$$

logo, temos uma equação do $2^{\circ}$ grau para $w$, cuja solução é dada por

$$
w=\frac{-i b \pm \sqrt{-b^{2}+4 m k}}{-2 m} .
$$

Na Eq. (7), podemos simplificar o termo dentro da raiz, de tal forma que, ao extrair a constante $b$, devido ao sinal menos, ela vem na forma de notação complexa, ou seja,

$$
w=\frac{i b}{2 m} \pm \frac{-i b}{2 m} \sqrt{1-\frac{4 m k}{b^{2}}}
$$

logo, temos dois valores de $w$ distintos. Substituindo esse resultado na Eq. (5), vem

$$
x_{h}(t)=A e^{\frac{-b t}{2 m}\left(1+\sqrt{1-\frac{4 m k}{b^{2}}}\right)}+B e^{\frac{-b t}{2 m}\left(1-\sqrt{1-\frac{4 m k}{b^{2}}}\right)} .
$$

Note que a notação complexa desaparece pelo fato de $i^{2}=-1$. A Eq. (9) possui dois coeficientes a serem determinados, logo, precisa de duas condições a serem impostas ao problema. Como o sistema evolui no tempo, podemos utilizar condições iniciais, tal que

$$
\left.x(t)\right|_{t=0}=\left.x_{o} \quad \therefore \quad \frac{d x(t)}{d t}\right|_{t=0}=v_{o}
$$

Agora faremos uma abordagem para verificar sob quais condições a solução dada pela Eq. (9) se comporta em função dos parâmetros do problema, ou seja, em função de $b, m$ e $k$. 


\subsection{Oscilador harmônico superamortecido}

Para este caso, como $b^{2}>4 m k$, logo, a expressão dentro da raiz da Eq. (9) é positiva. Desta forma, a solução não muda, o que implica que

$$
x_{h}(t)=A e^{\frac{-b t}{2 m}\left(1+\sqrt{1-\frac{4 m k}{b^{2}}}\right)}+B e^{\frac{-b t}{2 m}\left(1-\sqrt{1-\frac{4 m k}{b^{2}}}\right)} .
$$

Os coeficientes $A$ e $B$, podem ser determinados impondo as condições iniciais, dada pela Eq. 10. Para simplificar a notação, definiremos

$$
\begin{gathered}
w_{1} \equiv \frac{-b}{2 m}\left(1+\sqrt{1-\frac{4 m k}{b^{2}}}\right) \therefore \\
w_{2} \equiv \frac{-b}{2 m}\left(1-\sqrt{1-\frac{4 m k}{b^{2}}}\right)
\end{gathered}
$$

com isso, a Eq. (11) fica

$$
x_{h}(t)=A e^{w_{1} t}+B e^{w_{2} t} .
$$

A velocidade e aceleração do bloco poderão ser obtidas a partir da derivada da Eq. (12).

\subsection{Oscilador harmônico criticamente amortecido}

Para este caso, como $b^{2}=4 m k$, logo, temos duas raízes reais e iguais. Neste caso, a Eq. (9) torna-se,

$$
x_{h 1}(t)=A e^{-\frac{b t}{2 m}} .
$$

Sabemos que há diferentes funções que podem ser soluções de uma equação diferencial. Desta forma, uma combinação linear dessas funções também será solução. Devido a equação do oscilador harmônico amortecido ser expresso por uma derivada de segunda ordem, logo, possui duas condições iniciais, conforme mostra a Eq. 10. Se olharmos a equação acima, verificaremos que ela só satisfaz uma dessas condições. Então, podemos buscar uma nova função que também seja solução da Eq. (4), ou seja,

$$
x_{h 2}(t)=B t e^{-\frac{b t}{2 m}},
$$

onde uma combinação linear dessas soluções também será solução,

$$
x_{h}(t)=(A+B t) e^{-\frac{b t}{2 m}} .
$$

Semelhante ao que foi feito anteriormente, os coeficientes $A$ e $B$ podem ser determinados impondo as condições iniciais, dada pela Eq. (10). Para simplificar a notação, definiremos o parâmetro que indica a frequência de amortecimento do movimento da seguinte forma,

$$
\gamma \equiv-\frac{b}{2 m}
$$

com isso, a Eq. (13) fica

$$
x_{h}(t)=(A+B t) e^{\gamma t} .
$$

\subsection{Oscilador harmônico subamortecido}

Neste caso, como $b^{2}<4 m k$ torna-se necessário fazer algumas manipulações algébricas devido o termo da raiz da Eq. (9) assumir valores negativos. Para contorna este problema, podemos fazer uso da notação complexa $i$. Para simplificar a notação, usaremos a definição da frequência de amortecimento $\gamma$ e definiremos a frequência angular $w_{o}^{2} \equiv k / m$, da qual resulta

$$
\begin{array}{r}
\sqrt{1-\frac{4 m k}{b^{2}}}=\sqrt{1-\frac{4 m^{2} w_{o}^{2}}{b^{2}}}= \\
\sqrt{\frac{-4 m^{2}}{b^{2}}\left(w_{o}^{2}-\frac{b^{2}}{4 m^{2}}\right)}=-\frac{i}{\gamma} \sqrt{w_{o}^{2}-\gamma^{2}}
\end{array}
$$

Substituindo este resultado na Eq. (9) e fazendo algumas simplificações usando a equação de Euler, temos

$$
\begin{array}{r}
x_{h}(t)=A e^{\gamma t} \cos \left(\sqrt{w_{o}^{2}-\gamma^{2}} t\right)+ \\
B e^{\gamma t} \sin \left(\sqrt{w_{o}^{2}-\gamma^{2}} t\right) .
\end{array}
$$

Os coeficientes $A$ e $B$, podem ser determinados impondo as condições iniciais no problema. Para simplificar a notação, definiremos

$$
w \equiv \sqrt{w_{o}^{2}-\gamma^{2}}
$$

com isso, a Eq. 15 fica

$$
x_{h}(t)=A e^{\gamma t} \cos w t+B e^{\gamma t} \sin w t .
$$

Conforme mencionado anteriormente, a velocidade e aceleração serão obtidas a partir da derivada da Eq. (16). 


\subsection{Oscilador Harmônico Simples}

Neste caso, não há amortecimento e a energia do sistema é conservada. Como essa condição é uma simplificação do caso com amortecimento, então, podemos obter os resultados a partir dos casos citados anteriormente. Primeiramente, avaliaremos como a Eq. (9) se comporta para este caso. É fácil verificar que teremos divisão por zero no termo dentro da raiz, pois, neste caso $b=0$. Logo, esta equação, na forma como está escrita não permite obter uma expressão simplificada para o nosso caso.

Para o caso superamortecido, dado pela Eq. (11), podemos notar que não há nenhuma diferença em relação a Eq. (9). Logo, devemos descartar esta condição também. Para o caso criticamente amortecido, a solução dada pela Eq. (13) representa uma simplificação para resolução de equações diferenciais com raízes iguais [2], que não é o nosso caso. Para o caso subamortecido, a única consideração feita na Eq. (9) é que o termo dentro da raiz é negativo. A solução para este caso é dada pela Eq. (15). Para este caso, onde não há amortecimento, temos que $\gamma=0$. Desta forma, a Eq. 15 fica,

$$
x_{h}(t)=A \cos w_{o} t+B \sin w_{o} t .
$$

Podemos verificar se a energia do sistema é conservada. Para isso, precisamos calcular a taxa de variação da energia mecânica $E(t)$ no tempo. Usando a definição de energia cinética $E_{c}(t)$ e energia potencial $E_{p}(t)$, vem

$$
\begin{gathered}
E_{c}(t)=\frac{1}{2} m v(t)^{2} . \\
E_{p}(t)=\frac{1}{2} k x(t)^{2} . \\
E(t)=E_{c}(t)+E_{p}(t) .
\end{gathered}
$$

Derivando a energia total do sistema em relação ao tempo, temos

$$
\begin{array}{r}
\frac{d E(t)}{d t}=\frac{d E c(t)}{d t}+\frac{d E p(t)}{d t}= \\
v(t)(m a(t)+k x(t)),
\end{array}
$$

usando a Eq. (2), sem o termo de força externa, vem

$$
\frac{d E(t)}{d t}=-b v(t)^{2}
$$

integrando esta equação, temos que

$$
E(t)=E_{o}-b \int_{0}^{t} v(t)^{2} d t
$$

onde a integral quadrática da velocidade, representa a taxa de dissipação da energia em função da velocidade do bloco no tempo. Devido o termo quadrático da velocidade, a integral será sempre positiva, independente do valor da velocidade. Desta forma, a energia mecânica $E(t)$ sempre irá decrescer ao longo do tempo. Como assumimos que não há amortecimento, ou seja, $b=0$. Logo, a energia em qualquer instante de tempo será igual a energia inicial do sistema, tal que, $E(t)=E_{o}$.

\section{Oscilador harmônico amortecido forçado}

Conforme foi mencionado anteriormente, este é o caso mais geral para oscilações mecânicas, pois leva em conta a ação de forças dissipativas, bem como, forças externas atuando no sistema, que é representado pela Eq. (3). Devido as soluções para o caso homogêneo terem sido obtidas previamente, focaremos nossa atenção na obtenção da solução particular, cuja solução geral é dada pela combinação das duas soluções.

Definiremos a força externa $F(t)$ de modo a contemplar os seguintes casos:

$$
F(t)=F_{o} \cos \left(w^{\prime} t+\phi\right)\left\{\begin{array}{llll}
F_{o}, & \text { se } w^{\prime}=0 \text { e } \phi=0 \\
F_{o} \cos w^{\prime} t, & \text { se } w^{\prime} \neq 0 \text { e } \phi=0 \\
F_{o} \sin w^{\prime} t, & \text { se } w^{\prime} \neq 0 \text { e } \phi=\frac{3 \pi}{2} \\
F_{o}\left(\cos w^{\prime} t \pm \sin w^{\prime} t\right), & \text { se } w^{\prime} \neq 0 \text { e } \phi \neq n \frac{\pi}{2}, n=0,1,2, \ldots
\end{array}\right.
$$

Assim, podemos assumir que a solução para o caso particular seja

$$
x_{p}(t)=\bar{A} \cos w^{\prime} t+\bar{B} \sin w^{\prime} t,
$$

onde temos, 


$$
\frac{d x_{p}(t)}{d t}=-w^{\prime} \bar{A} \sin w^{\prime} t+w^{\prime} \bar{B} \cos w^{\prime} t
$$

e

$$
\frac{d^{2} x_{p}(t)}{d t^{2}}=-w^{\prime 2} \bar{A} \cos w^{\prime} t-w^{\prime 2} \bar{B} \sin w^{\prime} t
$$

Substituindo a solução para o caso particular e suas derivadas na Eq. (3), resulta

$$
\begin{array}{r}
-w^{\prime 2} \bar{A} \cos w^{\prime} t-w^{\prime 2} \bar{B} \sin w^{\prime} t+ \\
\frac{b}{m}\left(-w^{\prime} \bar{A} \sin w^{\prime} t+w^{\prime} \bar{B} \cos w^{\prime} t\right)+ \\
\frac{k}{m}\left(\bar{A} \cos w^{\prime} t+\bar{B} \sin w^{\prime} t\right)= \\
\frac{F_{o}}{m}\left(\cos w^{\prime} t \cos \phi-\sin w^{\prime} t \sin \phi\right) .
\end{array}
$$

Usando as definições de $w_{o}^{2}$ e $\gamma$ e comparando os termos para $\cos w^{\prime} t$ e $\sin w^{\prime} t$, respectivamente, vem

$\mathrm{e}$

$$
\bar{A}\left(w_{o}^{2}-w^{\prime 2}\right)-2 \gamma w^{\prime} \bar{B}=\frac{F_{o}}{m} \cos \phi,
$$

$$
\bar{B}\left(w_{o}^{2}-w^{\prime 2}\right)+2 \gamma w^{\prime} \bar{A}=-\frac{F_{o}}{m} \sin \phi .
$$

Resolvendo esse sistema, temos

$$
\bar{A}=\frac{F_{o}}{m} \cdot \frac{\left(w_{o}^{2}-w^{\prime 2}\right) \cos \phi-2 \gamma w^{\prime} \sin \phi}{\left(w_{o}^{2}-w^{\prime 2}\right)^{2}+4 \gamma^{2} w^{\prime 2}},
$$

e

$$
\bar{B}=-\frac{F_{o}}{m} \cdot \frac{2 \gamma w^{\prime} \cos \phi+\left(w_{o}^{2}-w^{\prime 2}\right) \sin \phi}{\left(w_{o}^{2}-w^{\prime 2}\right)^{2}+4 \gamma^{2} w^{\prime 2}},
$$

com isso, temos os coeficientes da solução particular, dada pela Eq. (23). Agora, podemos combinar a solução da equação homogênea com a solução da equação particular e obter assim a solução geral do oscilador mecânico.

\subsection{Oscilador harmônico superamortecido forçado}

Para este caso, temos que $b^{2}>4 m k$ e $F(t) \neq 0$. Combinando a solução da homogênea mais a particular, temos

$$
x(t)=A e^{w_{1} t}+B e^{w_{2} t}+\bar{A} \cos w^{\prime} t+\bar{B} \sin w^{\prime} t .
$$

Os coeficientes $A$ e $B$ serão obtidos impondo as condições iniciais do problema, dada pela Eq. 10 .
Note que estamos mantendo a mesma notação feita anteriormente e os coeficientes $\bar{A}$ e $\bar{B}$ já foram obtidos na solução do caso particular. Impondo as condições iniciais, temos

$$
\begin{gathered}
x_{o}=A+B+\bar{A} \\
v_{o}=w_{1} A+w_{2} B+w^{\prime} \bar{B}
\end{gathered}
$$

Resolvendo este sistema e substituindo na equação acima, temos

$$
\begin{gathered}
x(t)=\frac{\left(v_{o}-w^{\prime} \bar{B}\right)-\left(x_{o}-\bar{A}\right) w_{2}}{w_{1}-w_{2}} e^{w_{1} t}+ \\
\frac{\left(x_{o}-\bar{A}\right) w_{1}-\left(v_{o}-w^{\prime} \bar{B}\right)}{w_{1}-w_{2}} e^{w_{2} t}+ \\
\bar{A} \cos w^{\prime} t+\bar{B} \sin w^{\prime} t
\end{gathered}
$$

onde esta equação descreve o deslocamento do bloco em função da força de resistência do fluido e da força externa em função do tempo. Analogamente, podemos obter a velocidade $v(t)$, derivando a Eq. 29), da qual resulta,

$$
\begin{array}{r}
v(t)=\frac{\left(v_{o}-w^{\prime} \bar{B}\right)-\left(x_{o}-\bar{A}\right) w_{2}}{w_{1}-w_{2}} w_{1} e^{w_{1} t}+ \\
\frac{\left(x_{o}-\bar{A}\right) w_{1}-\left(v_{o}-w^{\prime} \bar{B}\right)}{w_{1}-w_{2}} w_{2} e^{w_{2} t}- \\
w^{\prime} \bar{A} \sin w^{\prime} t+w^{\prime} \bar{B} \cos w^{\prime} t
\end{array}
$$

com isso, temos uma expressa para a velocidade do bloco no sistema. Desta forma, a aceleração pela qual esse sistema está sujeito é dado por,

$$
\begin{array}{r}
a(t)=\frac{\left(v_{o}-w^{\prime} \bar{B}\right)-\left(x_{o}-\bar{A}\right) w_{2}}{w_{1}-w_{2}} w_{1}^{2} e^{w_{1} t}+ \\
\frac{\left(x_{o}-\bar{A}\right) w_{1}-\left(v_{o}-w^{\prime} \bar{B}\right)}{w_{1}-w_{2}} w_{2}^{2} e^{w_{2} t}- \\
w^{\prime 2} \bar{A} \cos w^{\prime} t-w^{\prime 2} \bar{B} \sin w^{\prime} t,
\end{array}
$$

As energias do sistema, tal como, a energia potencial $E_{p}(t)$ e cinética $E_{c}(t)$ podem ser obtidas em função das Eqs. (29) e (30), respectivamente. Combinando ambas, temos a energia total do sistema. O sistema retornaria para o estado de equilíbrio sem oscilar caso não houvesse forças externas atuando no sistema. Logo, a ação dessa força irá mudar o movimento do bloco. 


\subsection{Oscilador harmônico criticamente amortecido forçado}

Neste caso, temos que $b^{2}=4 m k$ e $F(t) \neq 0$. A solução geral para este caso é

$$
x(t)=(A+B t) e^{\gamma t}+\bar{A} \cos w^{\prime} t+\bar{B} \sin w^{\prime} t .
$$

Impondo as condições iniciais, temos

$$
\begin{gathered}
x_{o}=A+\bar{A} \\
v_{o}=B+\gamma A+w^{\prime} \bar{B}
\end{gathered}
$$

Resolvendo este sistema e substituindo na Eq. (32), temos

$$
\begin{aligned}
& x(t)=\left(\left(x_{o}-\bar{A}\right)(1-\gamma t)+\left(v_{o}-w^{\prime} \bar{B}\right) t\right) e^{\gamma t} \\
& +\bar{A} \cos w^{\prime} t+\bar{B} \sin w^{\prime} t .
\end{aligned}
$$

A velocidade $v(t)$ do bloco é dada por,

$$
\begin{aligned}
& \left.v(t)=\left(\left(v_{o}-w^{\prime} \bar{B}\right)(1+\gamma t)-\left(x_{o}-\bar{A}\right) \gamma^{2} t\right)\right) \\
& \times e^{\gamma t}-w^{\prime} \bar{A} \sin w^{\prime} t+w^{\prime} \bar{B} \cos w^{\prime} t
\end{aligned}
$$

com isso, temos uma expressa para a velocidade do bloco em função do tempo. Derivando a Eq. (34), temos que a aceleração é dada por,

$$
\begin{aligned}
& a(t)=\left(\left(v_{o}-w^{\prime} \bar{B}\right) \gamma(2+\gamma t)-\left(x_{o}-\bar{A}\right) \gamma^{2}\right. \\
& \times(1+\gamma t)) e^{\gamma t}-w^{\prime 2} \bar{A} \cos w^{\prime} t \\
& -w^{\prime 2} \bar{B} \sin w^{\prime} t
\end{aligned}
$$

Vale ressaltar que apesar de simplificarmos o sistema pelo fato de $b^{2}=4 m k$, isso não que dizer que o sistema não esteja sujeito a ação de forças dissipativas, pelo contrário, esta condição faz com que o sistema retorne mais rapidamente a condição de equilíbrio estável. É possível observar esse tipo de sistema em portas de escritórios de empresas que utilizam sistema de fechamento automático.

\subsection{Oscilador harmônico subamortecido forçado}

A solução geral para $b^{2}<4 m k$ e $F(t) \neq 0$ é dada pela soma da solução homogênea e particular, qual seja,

$$
\begin{array}{r}
x(t)=A e^{\gamma t} \cos w t+B e^{\gamma t} \sin w t+ \\
\bar{A} \cos w^{\prime} t+\bar{B} \sin w^{\prime} t,
\end{array}
$$

onde as notações e definições são as mesmas feitas anteriormente. Sendo assim, impondo as condições iniciais, temos

$$
\begin{gathered}
x_{o}=A+\bar{A} \\
v_{o}=\gamma A+w B+w^{\prime} \bar{B}
\end{gathered}
$$

Resolvendo este sistema, e substituindo na equação acima, temos

$$
\begin{array}{r}
x(t)=\left(x_{o}-\bar{A}\right) e^{\gamma t} \cos w t+ \\
\frac{\left(v_{o}-w^{\prime} \bar{B}\right)-\left(x_{o}-\bar{A}\right) \gamma}{w} \times \\
e^{\gamma t} \sin w t+\bar{A} \cos w^{\prime} t+\bar{B} \sin w^{\prime} t,
\end{array}
$$

onde esta equação descreve o deslocamento do bloco que será amortecido de forma mais lenta. Dependendo da intensidade da força externa, este sistema pode ter amortecimento desprezado devido a ação dessa força. A velocidade $v(t)$ pode ser obtida derivando a Eq. (37), ou seja,

$$
\begin{array}{r}
v(t)=\left(v_{o}-w^{\prime} \bar{B}\right) e^{\gamma t} \cos w t+ \\
\frac{\left(v_{o}-w^{\prime} \bar{B}\right) \gamma-\left(x_{o}-\bar{A}\right)\left(w^{2}+\gamma^{2}\right)}{w} \times \\
e^{\gamma t} \sin w t-w^{\prime} \bar{A} \sin w^{\prime} t+w^{\prime} \bar{B} \cos w^{\prime} t
\end{array}
$$

com isso, é possível expressar a velocidade do bloco em função do tempo. Sendo assim, a aceleração $a(t)$ é dada por,

$$
\begin{array}{r}
a(t)=\left(2 \gamma\left(v_{o}-w^{\prime} \bar{B}\right)-\left(x_{o}-\bar{A}\right)\left(w^{2}+\gamma^{2}\right)\right) \times \\
e^{\gamma t} \cos w t+\frac{\left(v_{o}-w^{\prime} \bar{B}\right)\left(\gamma^{2}-w^{2}\right)-\gamma\left(x_{o}-\bar{A}\right)\left(w^{2}+\gamma^{2}\right)}{w} \times \\
e^{\gamma t} \sin w t-w^{\prime 2} \bar{A} \cos w^{\prime} t-w^{\prime 2} \bar{B} \sin w^{\prime} t,
\end{array}
$$


Apesar do sistema ser fracamente amortecido, ele oscilará com frequência menor que o caso sem amortecimento $w<w_{o}$ e sua amplitude decresce exponencialmente no tempo, devido a frequência de amortecimento $\gamma$. O movimento desse bloco será influenciada pela ação da força externa atuando no sistema.

\subsection{Oscilador Harmônico Simples Forçado}

Neste caso, temos que $b=0$ e $F(t) \neq 0$. Esperase que por não haver forças de amortecimento, a energia do sistema se conserve. A posição do bloco é dada pela combinação da solução homogênea e particular, qual seja,

$$
\begin{array}{r}
x(t)=A \cos w_{o} t+B \sin w_{o} t+ \\
\bar{A} \cos w^{\prime} t+\bar{B} \sin w^{\prime} t .
\end{array}
$$

Os coeficientes $A$ e $B$, podem ser determinados impondo as condições iniciais no problema, tal que

$$
\begin{gathered}
x_{o}=A+\bar{A} \\
v_{o}=w_{o} B+w^{\prime} \bar{B}
\end{gathered}
$$

Resolvendo e substituindo na equação acima, temos

$$
\begin{array}{r}
x(t)=\left(x_{o}-\bar{A}\right) \cos w_{o} t+\frac{v_{o}-w^{\prime} \bar{B}}{w_{o}} \sin w_{o} t+ \\
\bar{A} \cos w^{\prime} t+\bar{B} \sin w^{\prime} t,
\end{array}
$$

onde esta equação descreve o deslocamento do bloco para o caso sem amortecimento. Devido a ação da força externa, a amplitude do sistema pode ser alterada em função da intensidade dessa força. Como feito anteriormente, a velocidade $v(t)$ pode ser obtida derivando a equação acima, da qual resulta,

$$
\begin{array}{r}
v(t)=\left(v_{o}-w^{\prime} \bar{B}\right) \cos w_{o} t-\left(x_{o}-\bar{A}\right) w_{o} \sin w_{o} t- \\
w^{\prime} \bar{A} \sin w^{\prime} t+w^{\prime} \bar{B} \cos w^{\prime} t
\end{array}
$$

com isso, é possível expressar a velocidade do bloco em função do tempo. Logo, a aceleração $a(t)$ é dada por,

$$
\begin{array}{r}
a(t)=-\left(x_{o}-\bar{A}\right) w_{o}^{2} \cos w_{o} t-\left(v_{o}-\right. \\
\left.w^{\prime} \bar{B}\right) w_{o} \sin w_{o} t-w^{\prime 2} \bar{A} \cos w^{\prime} t-w^{\prime 2} \bar{B} \sin w^{\prime} t .
\end{array}
$$

Os estudos sobre oscilações forçadas ganharam grande interesse devido aos fenômenos de ressonância, dos quais as amplitudes assumem valores significativos resultando na destruição de prédios e pontes após entrarem em ressonância devido a ação de forças externas, tais como, ventos ou terremotos. Esses fenômenos são provenientes dos denominadores das Eqs. 26) e 27) quando a frequência imposta pela força externa se aproxima consideravelmente da frequência natural $w^{\prime} \simeq w_{o}$. Se analisarmos essas equações, podemos notar que devido aos termos quadráticos do denominador, mesmo para valores de $w^{\prime}>w_{o}$ e $\gamma^{2}>0$, não teremos divisão por zero. No entanto, é possível encontrar valores de $w^{\prime}=w_{o} \pm f\left(\gamma, w_{o}\right)$, que façam com que esses denominadores assumam valores próximos de zero. Para isso, temos que

$$
\frac{d \bar{A}}{d w^{\prime}}=0 \quad \therefore \quad \frac{d \bar{B}}{d w^{\prime}}=0
$$

com isso, encontramos os valores de $w^{\prime}$ para os quais, as amplitudes do sistema sejam máximas resultando em fenômenos de ressonância.

Agora, verificaremos se a energia é conservada para o caso de forças externas atuando no sistema. Combinando as Eqs. (21) e (2), temos que a taxa de variação da energia total do sistema é,

$$
\frac{d E(t)}{d t}=F(t) v(t)-b v(t)^{2},
$$

integrando esta equação, vem

$$
E(t)=E_{o}+\int_{0}^{t} F(t) v(t) d t-b \int_{0}^{t} v(t)^{2} d t,
$$

onde temos o termo que representa a taxa de dissipação da energia em função da velocidade quadrática do bloco e o termo de fonte externa que contribui para o aumento da energia no sistema. Se assumirmos que não há forças dissipativas no sistema, podemos observar que a energia não é conservada devido a força externa. Com isso, energia mecânica $E(t)$ mudará ao longo do tempo. Logo, mesmo que não haja nenhuma força dissipativa atuando no sistema, isso não quer dizer que a energia do sistema se conserve. No entanto, pela Eq. (44) podemos obter uma relação que permita que a energia se conserve mesmo sob a ação de forças externas e dissipativas, tal que,

$$
F(t)=b v(t)
$$




\section{Solução numérica do oscilador harmônico amortecido forçado}

O método proposto para discretizar essa equação é conhecido como método de diferenças finitas, no qual o intervalo de tempo proposto para a solução numérica implica em um $\Delta t$ na ordem $\left(10^{-4}\right.$ à $10^{-1} \mathrm{~s}$ ). A precisão do método está diretamente relacionado com o intervalo de tempo.

Para isso, usaremos o operador de diferenças avançadas de primeira ordem, que visa aproximar a derivada da velocidade em função do tempo, a partir de dois pontos consecutivos. Sendo assim, podemos reduzir a ordem da derivada da Eq. (3), de $2^{a}$ ordem da posição no tempo, para uma equação de $1^{a}$ ordem para velocidade. Usando a definição dos parâmetros $w_{o}$ e $\gamma$, temos

$$
\frac{d v(t)}{d t}=\frac{F(t)}{m}+2 \gamma v(t)-w_{o}^{2} x(t) .
$$

Como podemos notar, a derivada da velocidade pode ser representado por uma função que depende da posição, velocidade, força e do tempo, isto é,

$$
\frac{d v(t)}{d t}=f(x, v, F, t)
$$

Através do método de diferenças finitas, podemos calcular aproximadamente a derivada da velocidade $v(t)[3]$ no intervalo $[t, t+\Delta t]$ da seguinte maneira,

$$
\frac{d v(t)}{d t}=\frac{v(t+\Delta t)-v(t)}{\Delta t}
$$

onde aproxima a função $v(t)$ que passa pelos instantes $t$ e $t+\Delta t$, com um erro de truncamento na ordem do intervalo de tempo, isto é, $O(\Delta t)$. Por isso, quando menor for o intervalo de tempo, consequentemente, o erro será menor possível, fazendo com que a solução numérica tenda a se aproximar consideravelmente da solução analítica. Porém, isso requer um esforço computacional bastante significativo, dependendo da precisão desejada.

Simplificando a notação, podemos definir $v_{t} \equiv$ $v(t)$ e $v_{t+1} \equiv v(t+\Delta t)$. Semelhantemente, temos $x_{t} \equiv x(t)$ e $F_{t} \equiv F(t)$. Usando essas definições e substituindo na Eq. (47), temos

$$
\frac{v_{t+1}-v_{t}}{\Delta t}=\frac{F_{t}}{m}+2 \gamma v_{t}-w_{o}^{2} x_{t},
$$

resultando em

$$
v_{t+1}=v_{t}(1+2 \gamma \Delta t)+\frac{F_{t}}{m} \Delta t-w_{o}^{2} x_{t} \Delta t
$$

onde esta equação permite calcular a velocidade a cada intervalo de tempo em função dos parâmetros do problema no instante anterior. Analogamente, podemos determinar a posição do bloco em função da velocidade, tal que,

$$
v(t)=\frac{d x(t)}{d t}=\frac{x(t+\Delta t)-x(t)}{\Delta t},
$$

logo, fazendo uso das notações definidas anteriormente, vem

$$
x_{t+1}=x_{t}+v_{t} \Delta t,
$$

onde a posição do bloco será calculada em função de sua velocidade, dada pela Eq. (50). Da mesma forma, temos que a aceleração é dada por

$$
a(t)=\frac{d v(t)}{d t}=\frac{v(t+\Delta t)-v(t)}{\Delta t},
$$

resultando em,

$$
a_{t}=\frac{v_{t+1}-v_{t}}{\Delta t} .
$$

É fácil notar que a Eq. 50 representa a solução do oscilador harmônico amortecido forçado para a velocidade do bloco em função do tempo. Através da velocidade, podemos determinar a posição usando a Eq. (51) e determinar a aceleração em função da derivada da velocidade usando apenas a aproximação por diferenças. Com isso, podemos determinar a posição, velocidade, aceleração e energia usando o método numérico de diferenças finitas. Conforme definido anteriormente, as energias do sistema serão obtidas pelas Eqs. (18), 19) e (20). As simplificações na solução numérica serão expressas em função dos dados do problema. Para ilustrar, para o caso em que não há forças dissipativas atuando no sistema, temos que $\gamma=0$ na Eq. (50). Semelhantemente, para o caso de não haver forças externas atuando no sistema, implica que $F_{t}=0$. Como a posição e aceleração são obtidas a partir da Eq. (50), logo, serão influenciados por essas simplificações.

Para ilustrar, no instante $t_{o}$, o bloco não se deslocou e está localizada na posição inicial $x_{o}$. Como o intervalo de tempo é nulo, logo, a velocidade tem que ser a velocidade inicial $v_{o}$ do sistema. Visto isso, cabe a pergunta: Qual é a aceleração do sistema? Segundo as soluções analíticas para os diferentes casos, assumindo que $t_{o}=0$, a aceleração não é nula. Isso pode ser observado pelas Eqs. (31), (35), (39) e 43). Este é o ponto em que a interpretação da solução analítica difere da solução numérica. Pela 
Eq. (52), a aceleração é obtida pela taxa de variação da velocidade no tempo. Em $t_{o}$ essa variação é nula, logo, resultando numa aceleração nula. Com isso, podemos observar que a condição inicial para aceleração do caso analítico difere do caso numérico. No entanto, as energias do sistema por não dependerem da aceleração permanecem iguais. Para $t_{1}$, a partícula evoluiu no tempo em um intervalo $\Delta t$, tal que, $t_{1}=t_{o}+\Delta t$. Desta forma, sua velocidade $v_{1}$ é obtido pela Eq. (50), a posição $x_{1}$ é calculada pela Eq. (51) e a aceleração $a_{0}$ pela diferença da velocidade, dada pela Eq. (52). As energias do sistema serão calculadas em função da posição e velocidade do bloco. O intervalo de tempo pode ser computado como $t_{i}=t_{o}+i \Delta t$, com $i=0, \ldots, n$.

\section{Resultados}

Apresentaremos os resultados obtidos pela solução analítica e compararemos com os resultados obtidos pelo método numérico para validar a metodologia implementada neste artigo.

O simulador de oscilações mecânicas foi desenvolvido usando a plataforma microsoft visual studio ultimate 2012 através da linguagem de programação visual basic.net [4]. O grande diferencial deste simulador é que ele permite acompanhar as mudanças nas variáveis de interesse ao longo do tempo através da exibição de dados na tela, bem como, através da visualização gráfica. A Fig. 2 mostra a tela do simulador.

Como podemos observar, há duas opções de cálculo usando soluções analíticas e numéricas e os dados do problema são tais que, $m(\mathrm{~kg})$ é a massa do bloco, $k(\mathrm{~N} / \mathrm{m})$ é a constante elástica da mola, $b(\mathrm{~kg} / \mathrm{s})$ é a constante de amortecimento devido a força de resistência do fluido, $t$ (s) é o tempo de simulação, $\Delta t(\mathrm{~s})$ o intervalo de tempo aplicado à solução numérica ${ }^{2}, x_{0}(\mathrm{~m})$ é a posição inicial do bloco, $v_{0}(\mathrm{~m} / \mathrm{s})$ é a velocidade inicial do bloco, $F_{0}$ $(\mathrm{N})$ é a força externa inicial que atua no sistema, $w^{\prime}(\mathrm{rad} / \mathrm{s})$ é a frequência angular e $\phi(\mathrm{rad} / \mathrm{s})$ é o ângulo de fase inicial que é expresso por um ângulo múltiplo de $\pi$.

Dependendo das variáveis do problema, ou seja, $m, k, b$ e $F_{o}$, o tipo de sistema analisado será exibido no canto esquerdo inferior da tela do simulador. $\mathrm{O}$ simulador permite escolher as variáveis de interesse a ser exibida graficamente, bem como, a opção de interromper e pausar a simulação. Dúvidas e sugestões podem ser encaminhadas para o email de contato que é exibido no canto direito inferior do simulador, passando o mouse sobre projeto física para todos.

As equações obtidas neste artigo estão presentes em várias literaturas que abordam oscilações mecânicas [5, 6]. Para ilustrar, considere um oscilador harmônico simple forçado e que o ângulo de fase $\phi=0$. Isto implica que, pela Eq. (27), temos que $\bar{B}=0$. Impondo as condições iniciais, tais que, o bloco esteja na origem e em repouso em $t=0$, a Eq. 41) fica,

$$
x(t)=\frac{F_{o}}{m} \cdot \frac{\cos w^{\prime} t-\cos w_{o} t}{w_{o}^{2}-w^{\prime 2}},
$$

onde está equação está de acordo com a literatura [5]. Podemos observar que para $w^{\prime} \simeq w_{o}$, teremos uma indeterminação na Eq. (53). Para contornar isso, podemos usar a regra de l'Hôpital [7], isto é, derivando o numerador e denominador em relação $w^{\prime}$. Aplicando o limite quando $w^{\prime} \rightarrow w_{o}$, temos,

$$
x(t)=\frac{F_{o}}{2 m w_{o}} t \sin w_{o} t .
$$

A Fig. 3 mostra os resultados obtidos pela Eq. (53) para $w^{\prime} \simeq w_{o}$. Como podemos observar, o deslocamento do bloco cresce em função do tempo contribuindo para o aumento da energia total do sistema. Graficamente é fácil verificar quando um sistema evolui para um fenômeno de ressonância, basta apenas monitorar sua amplitude de oscilação. Se essa amplitude cresce em função do tempo, esse sistema entrará em colapso.

Os fenômenos de ressonância estão diretamente relacionados a ação da força externa, ou seja, relacionados a solução particular do oscilador harmônico. Na literatura [6], encontramos apenas uma frequência de ressonância, dada por $w_{r}=\sqrt{w_{o}^{2}-2 \gamma^{2}}$ que permite o coeficiente da solução particular assumir valores máximos. Neste caso, a solução é dada por,

$$
x_{p}(t)=\frac{F_{o}}{m} \cdot \frac{\cos \left(w^{\prime} t-\delta\right)}{\sqrt{\left(w_{o}^{2}-w^{\prime 2}\right)^{2}+4 \gamma^{2} w^{\prime 2}}},
$$

onde o ângulo de fase $\delta=\arctan \frac{-2 \gamma w^{\prime}}{w_{a}^{2}-w^{\prime 2}}$.

A solução particular apresentada neste artigo, dada pela Eq. (23), pode ser escrita na mesma forma

\footnotetext{
${ }^{2}$ Apesar da solução analítica não depender desta variável, recomenda-se que utilize um passo $\Delta t=10^{-1}$ para visualização gráfica do oscilador. O intervalo de tempo para solução numérica é da ordem de $\Delta t=10^{-4}$ à $10^{-1}$.
} 


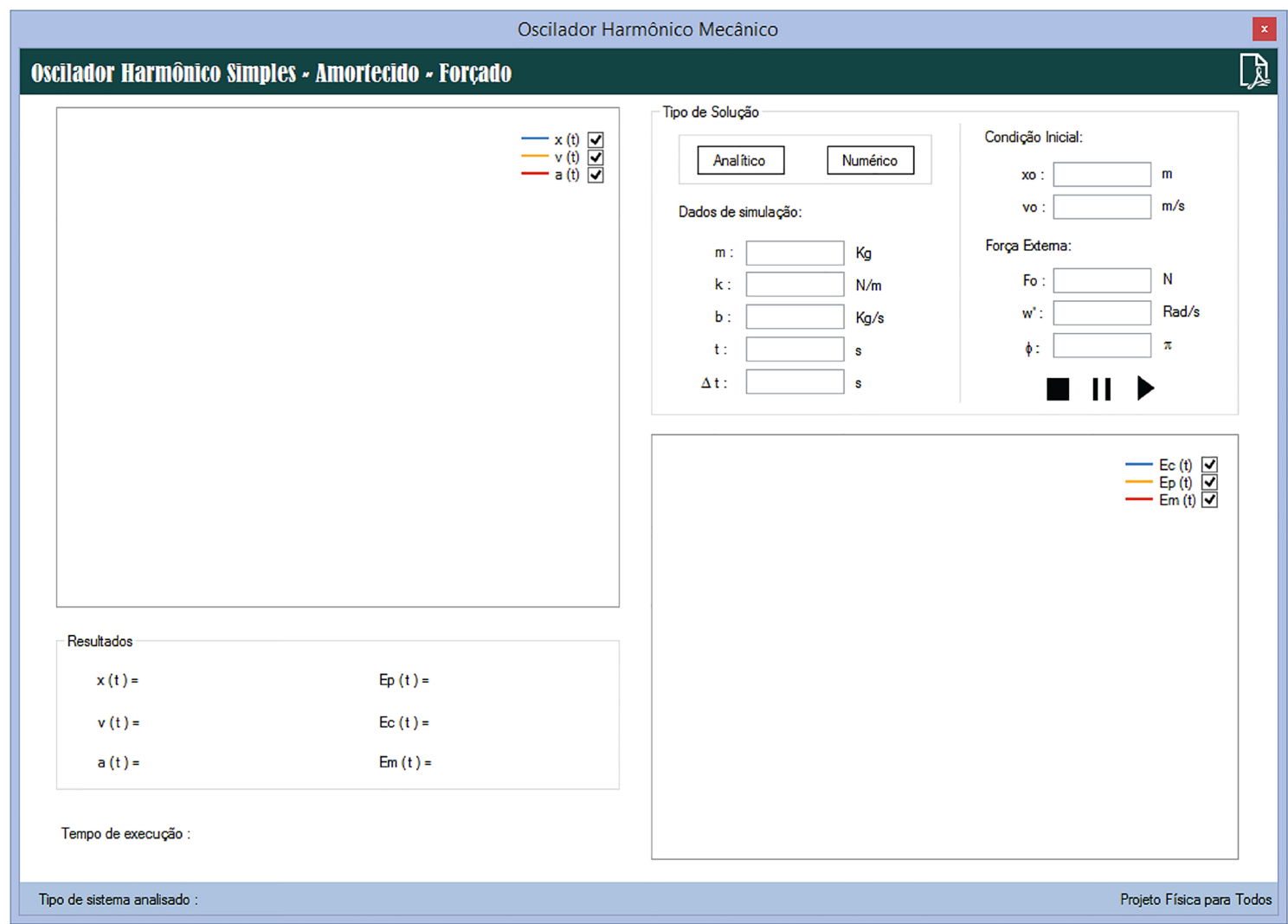

Figura 2: Simulador de oscilações mecânicas.

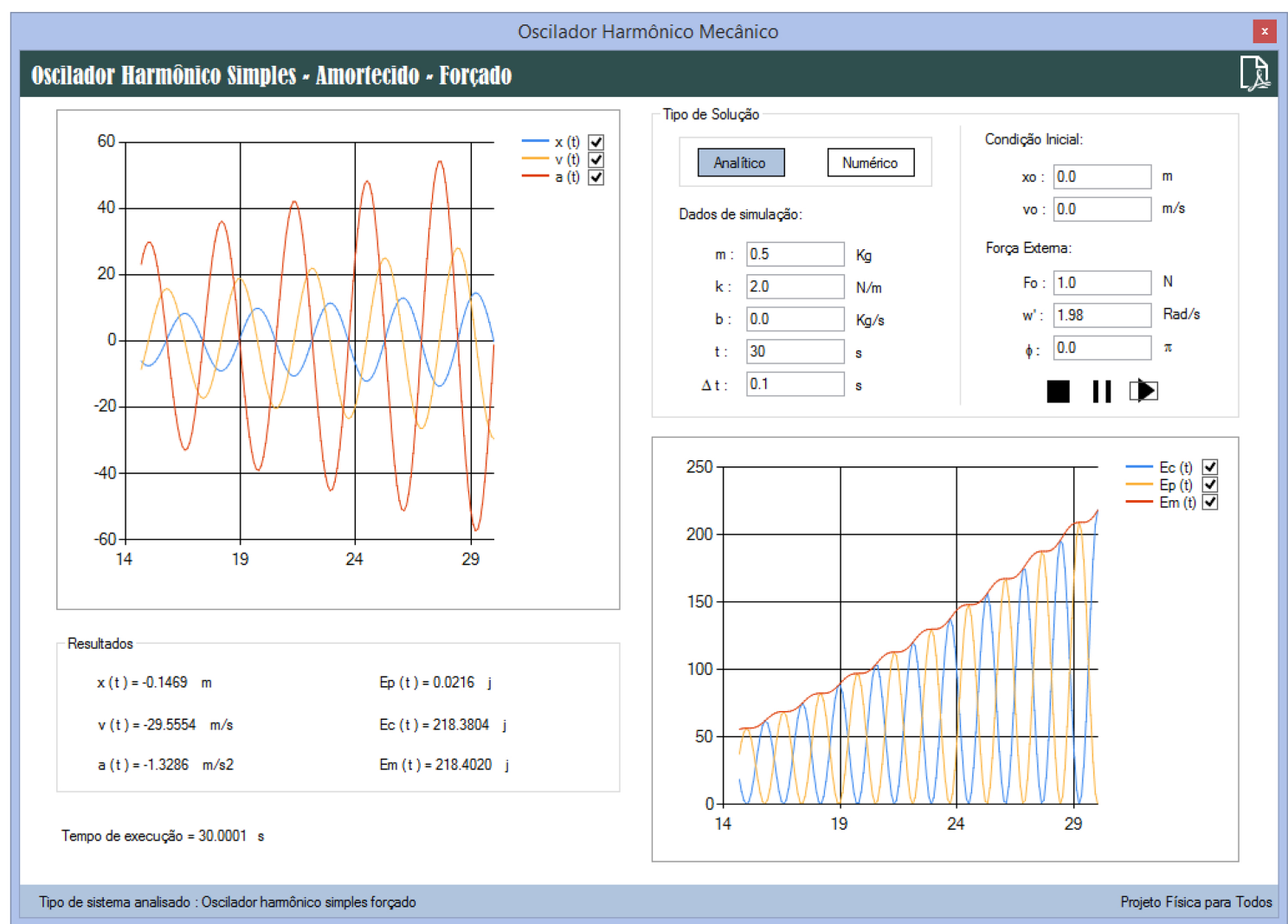

Figura 3: Ressonância para oscilações simples e forçada. 
da Eq. (55). Para isso, basta assumir que $\phi=0$ e definir as relações trigonométricas,

$$
\begin{aligned}
\cos \delta & \equiv \frac{w_{o}^{2}-w^{\prime 2}}{\sqrt{\left(w_{o}^{2}-w^{\prime 2}\right)^{2}+4 \gamma^{2} w^{\prime 2}}} \\
\sin \delta & \equiv-\frac{2 \gamma w^{\prime}}{\sqrt{\left(w_{o}^{2}-w^{\prime 2}\right)^{2}+4 \gamma^{2} w^{\prime 2}}} .
\end{aligned}
$$

A razão de não usarmos essas definições [5, 6] é que ela nos limita a encontra apenas uma condição para frequência de ressonância $w_{r}$. Agora, usando a solução particular dada pela Eq. (23), cujos coeficientes são dados pelas Eqs. (26) e (27), os pontos de máximos, por exemplo para $A$, resulta num polinômio de grau 5, dado por,

$$
A w^{\prime 5}+B w^{\prime 4}+C w^{\prime 3}+D w^{\prime 2}+E w^{\prime}+F=0,
$$

onde $A=\cos \phi, B=3 \gamma \sin \phi, C=-2 w_{o}^{2} \cos \phi$, $D=2 \gamma \sin \phi\left(2 \gamma^{2}-w_{o}^{2}\right), E=w_{o}^{2} \cos \phi\left(w_{o}^{2}-4 \gamma^{2}\right) \mathrm{e}$ $F=-\gamma w_{o}^{4} \sin \phi$. As raízes desse polinômio resultaram em cinco condições das quais o coeficiente $\bar{A}$ assume valores máximo $3^{3}$. Analogamente, podemos proceder da mesma forma para $\bar{B}$. Os valores máximos desses coeficientes podem resultar em fenômenos de ressonância desde que ultrapassem os limites estabelecidos pelo sistema analisado. Outro ponto importante a destacar é que as raízes da Eq. (56) estão diretamente relacionados com o ângulo de fase $\phi$ da força externa. Para ilustrar consideremos os seguintes casos:

i) $F(t)=F_{o} \cos \left(w^{\prime} t\right)$ : Neste caso, temos que $\phi=0$. Logo, a Eq. (56) fica,

$$
w^{\prime 5}-2 w_{o}^{2} w^{\prime 3}+w_{o}^{2}\left(w_{o}^{2}-4 \gamma^{2}\right) w^{\prime}=0,
$$

$$
\text { cujas raízes são: }\left[0, \pm \sqrt{w_{o}^{2}+2 \gamma w_{o}},\right.
$$
$\pm \sqrt{w_{o}^{2}-2 \gamma w_{o}}$.

ii) $F(t)=F_{o} \sin \left(w^{\prime} t\right)$ : Neste caso, temos que $\phi=\frac{3 \pi}{2}$. Com isso, a Eq. 56 fica,

$$
-3 \gamma w^{4}-2 \gamma\left(2 \gamma^{2}-w_{o}^{2}\right) w^{\prime 2}+\gamma w_{o}^{4}=0,
$$

cujas raízes são:

$$
\begin{aligned}
& {\left[ \pm \frac{1}{3} \sqrt{3 w_{o}^{2}-6 \gamma^{2}+6 \sqrt{w_{o}^{4}-\gamma^{2} w_{o}^{2}+\gamma^{4}}}\right.} \\
& \left. \pm \frac{1}{3} \sqrt{3 w_{o}^{2}-6 \gamma^{2}-6 \sqrt{w_{o}^{4}-\gamma^{2} w_{o}^{2}+\gamma^{4}}}\right]
\end{aligned}
$$

É fácil verificar que as raízes de $w^{\prime}$ que permitem o coeficiente $\bar{A}$ assumir valores máximos para $\phi=0$, correspondem as mesmas raízes que permitem o coeficiente $\bar{B}$ assumir valores máximos para $\phi=\frac{3 \pi}{2} \mathrm{e}$ vice-versa.

A Fig. 4 mostra os resultados para o oscilador harmônico subamortecido forçado para diferentes frequências de ressonância obtidas acima em função do ângulo de fase $\phi$. Os dados usados nesse teste foram: $m=2 \mathrm{~kg}, k=10 \mathrm{~N} / \mathrm{m}, b=1.5 \mathrm{~kg} / \mathrm{s}, F_{o}=10 \mathrm{~N}$ e $x_{o}=v_{o}=0$. Como podemos observar, as amplitudes de oscilações são diferentes para cada frequência de ressonância associada as raízes da Eq. (56). Note que não há a possibilidade de generalizar esses resultados, uma vez que, o grau do polinômio da Eq. (56) muda em função do ângulo de fase $\phi$ associado a força externa. As raízes $w_{r}$ obtidas pela Eq. (56), mesmo para $\phi=0$, diferem da frequência de ressonância $w_{r}$ da literatura. Isso ocorre porque parte do coeficiente da Eq. (55) está oculto no ângulo de fase $\delta$ da solução particular.

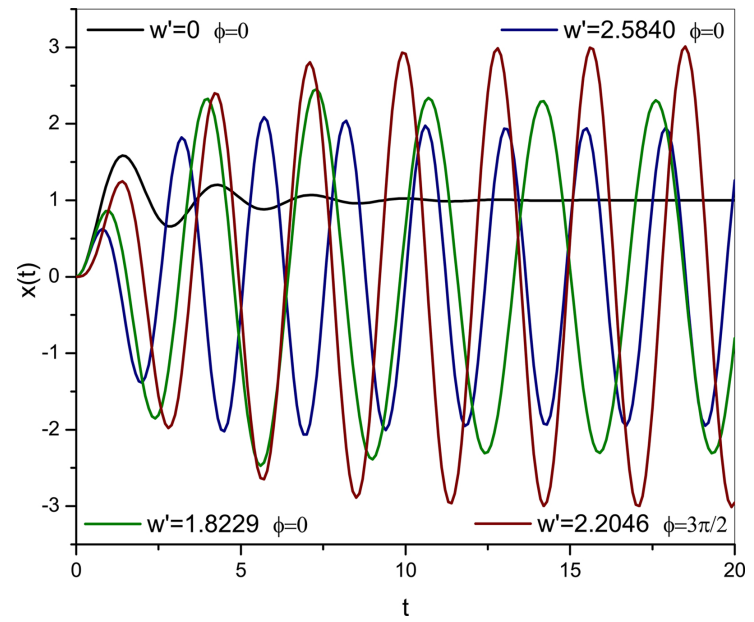

Figura 4: Posição do bloco para diferentes frequências de ressonância.

Outro ponto importante a destacar é que esse sistema oscilaria com amplitude decrescente no tempo até atingir o repouso devido as forças dissipativas. A Fig. 4 mostra que a amplitude de oscilação crescer até atingir seu valor máximo. Isso ocorre porque a força externa supera a força dissipativa e o sistema passa a oscilar com amplitude máxima para $t \geq 10 \mathrm{~s}$. Retomemos a nossa atenção na validação do método numérico implementado neste artigo. Para isso, apresentaremos os resultados obtidos pela solução

${ }^{3}$ Vale ressaltar que a condição para obtenção de pontos de máximos é a mesma para pontos de mínimos, isto é, que sua derivada seja nula. 
analítica e compararemos com os resultados obtidos numericamente para diferentes intervalos de tempo, visando mostrar sob quais condições o método de diferenças finitas deve ser aplicado. As Tabelas 1 e 2 mostram as comparações dos resultados obtidos pela solução analítica e numérica para dois casos distintos e diferentes intervalos de tempo, bem como, o erro relativo percentual que é dado por,

$$
\operatorname{Erro}(\%)=\left|\frac{R_{a}-R_{n}}{R_{a}}\right| \times 100 \%,
$$

onde $R_{a}$ e $R_{n}$ representam, respectivamente, os resultados analíticos e numéricos.

Como podemos observar nos resultados mostrados nas Tabelas 1 e 2, o método de diferenças finitas se torna preciso quanto menor for o intervalo de tempo $\Delta t$. Isso pode ser verificado, a partir dos erros relativos percentuais apresentados nessas tabelas.

\section{Conclusões}

Usando o método de diferenças finitas obtivemos três equações que contemplam todos os casos citados anteriormente, tais como: oscilador harmônico simples forçado, oscilador harmônico amortecido (subamortecido, criticamente amortecido e superamortecido) forçado, dos quais, geraram várias equações para cada caso resolvido analiticamente. Um outro ponto a destacar em relação ao método de diferenças finitas é que nem sempre é possível obter soluções analíticas e quando isso é possível, geralmente é feito algu- mas aproximações para simplificar o problema em questão. Logo, devemos destacar que o mesmo não ocorre para soluções numéricas. Neste artigo, buscamos mostrar uma implementação bastante simples de um método numérico visando resolver problemas aplicados à física. Usando o método de diferenças finitas podemos obter resultados mais precisos usando intervalo de tempo menores, tal como, $\Delta t \leq 10^{-5} \mathrm{~s}$, ou podemos usar outros métodos de solução, tal como, Crank-Nicholson, Runge-Kutta [8] entre outros.

O objetivo desse artigo foi mostrar uma física bastante simples e as possíveis abordagens ao resolver esse tipo de problema. O simulador de oscilações mecânicas visa mostrar o comportamento das variáveis de interesse sem a necessidade de resolver nenhuma equação. Pela análise de dados gerados na tela do simulador, bem como, pela visualização gráfica é possível verificar, por exemplo, sob quais condições a energia do sistema é conservada. Um mito, leva muitos leitores a acharem que a energia do sistema só é conservada quando não há forças dissipativas atuando sobre ele. Pela simulação para o caso de forças dissipativas nulas e forças externas diferente de zero, é possível observar que a energia do sistema não é conservada. Isso despertará o interesse em descobrir o porquê que isso acontece. Sendo assim, o interesse em buscar uma formulação que justifique tal fato, despertará seu interesse em física. Esse é um dos objetivos desse artigo.

Tabela 1: Resultados para oscilador harmônico subamortecido - $\Delta t=10^{-3}$

\begin{tabular}{|c|c|c|c|c|c|c|}
\hline Tempo (s) & $x_{a}(t)^{4}(m)$ & $x_{n}(t)^{5}(m)$ & $\operatorname{Erro}(\%)$ & $v_{a}(t)(m / s)$ & $v_{n}(t)(m / s)$ & $\operatorname{Erro}(\%)$ \\
\hline 1 & -2.3393 & -2.3559 & 0.7 & 35.5593 & 35.9231 & 1.0 \\
\hline 3 & 3.8593 & 3.9659 & 2.76 & -18.2389 & -18.8362 & 3.27 \\
\hline 5 & -3.5154 & -3.6839 & 4.79 & 5.6029 & 6.0310 & 7.64 \\
\hline 8 & -0.8458 & -0.8930 & 5.58 & 8.3696 & 9.1038 & 8.77 \\
\hline 10 & 1.0788 & 1.1840 & 9.75 & -3.8766 & -4.3270 & 11.62 \\
\hline
\end{tabular}

Tabela 2: Resultados para oscilador harmônico simples forçado - $\Delta t=10^{-4}$

\begin{tabular}{ccccccc}
\hline Tempo $(\mathrm{s})$ & $x_{a}(t)(m)$ & $x_{n}(t)(m)$ & $\operatorname{Erro}(\%)$ & $v_{a}(t)(\mathrm{m} / \mathrm{s})$ & $v_{n}(t)(\mathrm{m} / \mathrm{s})$ & $\operatorname{Erro}(\%)$ \\
\hline 1 & 0.4590 & 0.4590 & 0 & 0.0520 & 0.0522 & 0.38 \\
5 & -1.2593 & -1.2596 & 0.024 & -4.5759 & -4.5788 & 0.063 \\
10 & 4.3528 & 4.3280 & 0.57 & 5.3974 & 5.4872 & 1.66 \\
20 & 8.6129 & 8.4154 & 2.29 & -9.6215 & -10.3918 & 8.0 \\
\hline \multicolumn{7}{c}{$m=0.5 \mathrm{~kg}, k=2 \mathrm{~N} / \mathrm{m}, x_{o}=v_{o}=0, F_{o}=1 \mathrm{~N}, \mathrm{w}^{\prime}=1.98 \mathrm{rad} / \mathrm{s}, \phi=0$}
\end{tabular}




\section{Referências}

[1] A.C.M. Alvim, Métodos Numéricos em Engenharia Nuclear (Editora Certa, 2007), $1^{\mathrm{a}}$ ed.

[2] K.D. Machado, Equações Diferenciais Aplicadas Volume 1 (Editora Toda Palavra, 2012), $1^{\underline{a}}$ ed.

[3] C. Scherer, Métodos Computacionais da Física (Editora Livraria da Física, 2010), $2^{\mathrm{a}}$ ed.

[4] M. Halvorson, Microsoft Visual Basic 2013 - Passo a Passo (Editora Bookman, 2015), $1^{\mathrm{a}}$ ed.
[5] H.M. Nussenzveig, Fúsica Básica - Volume 2 (Editora Edgard Blucher, 1996), $3^{\mathrm{a}}$ ed.

[6] S.T. Thornton and J.B. Marion, Classical Dynamics of Particles and Systems (Academic Press, New York, 2004), 5th ed.

[7] L. Leithold, O Cálculo com Geometria Analítica (Editora Harbra, 1994), $3^{\mathrm{a}}$ ed.

[8] S. Nakamura, Computational Method in Engineering and Science (John Wiley and Sons, 1977), 1st ed. 